\title{
Endoventricular Circular Patch Plasty With Aortic Valve Replacement for Post-Infarction Cardiac Rupture Complicated With Aortic Valve Stenosis Case Report
}

\author{
Masahiro Ikeda, MD; Hirokazu Ohashi, MD; Yasushi Tsutsumi, MD; \\ Takahiro Kawai, MD; Masateru Ohnaka, MD
}

\begin{abstract}
Cardiac rupture after acute myocardial infarction (AMI) in patients with aortic valve stenosis (AS) is uncommon and only 2 survival cases after surgery have been reported to date. The present patient, a 69-year-old woman with aortic valve stenosis (AS), suffered an acute myocardial infarction and despite a successful baloon angioplasty to the proximal left anterior descending artery, the condition progressed into congestive heart failure. Echocardiography demonstrated AS with a valve area of $0.7 \mathrm{~cm}^{2}$ and a left ventricular aneurysm with a thin and dyskinetic anterior to apical wall. An urgent operation was performed and the opening of the pericardium revealed oozing rupture of the aneurysm. An endoventricular circular patch plasty combined with aortic valve replacement was successfully performed, and good results during 2-year follow-up were achieved. Physiological repair with exclusion of the infarcted area is essential to achieve a good long-term outcome in such a critical case. Special care should be taken with AMI in a patient with AS because of the possible occurrence of aneurysm and rupture of the left ventricle. (Circ J 2002; 66: 974-976)
\end{abstract}

Key Words: Aortic valve stenosis; Cardiac rupture; Endoventricular circular patch plasty; Myocardial infarction

A 69-year-old woman with aortic valve stenosis (AS) suffered an acute myocardial infarction (AMI), the first event resulting from total occlusion of the proximal left anterior descending coronary artery. Although successful percutaneus transluminal balloon angioplasty was performed urgently, the condition progressed into congestive heart failure and required respiratory control with a mechanical ventilator for 5 days. Twenty days after the angioplasty, the heart failure deteriorated and the patient was transferred for surgical treatment in New York Heart Association (NYHA) functional class IV. Echocardiography demonstrated AS with valve area of $0.7 \mathrm{~cm}^{2}$, a pressure gradient across the valve of $70 \mathrm{mmHg}$, and severe tricupsid valve regurgitation. A left ventricular aneurysm with a thin and dyskinetic anterior to apical wall was found (Fig 1). No findings of pericardial effusion were found. The preoperative hemodynamic data are shown in Table 1 . An urgent operation was performed. The opening of the pericardium revealed oozing rupture of the left ventricular aneurysm with a small amount of bloody pericardial effusion. Total hypothermic cardiopulmonary bypass was initiated, and the heart was arrested with cold blood cardioplegia. The left ventricle was incised at the apical portion of the aneurysm. An intraventricular thrombus was found and removed. The aortic valve was severely calcified, and we replaced it with a 19-mm St Jude mechanical prosthesis. Following closure of

(Received June 13, 2001; revised manuscript received August 20, 2001; accepted September 19, 2001)

Department of Cardiovascular Surgery, Fukui Cardiovascular Center, Fukui, Japan

Mailing address: Masahiro Ikeda, MD, Department of Cardiovascular Surgery, Toyama Redcross hospital, Ushijimahonmachi 2-1-58, Toyama 930-0859, Japan. E-mail: ikeda@toyama-med.jrc.or.jp the aortomy, circumferencial purse-string suturing of the endomyocardium was performed at the borderline of the infarcted and noninfarcted myocardium, and then a pericar-

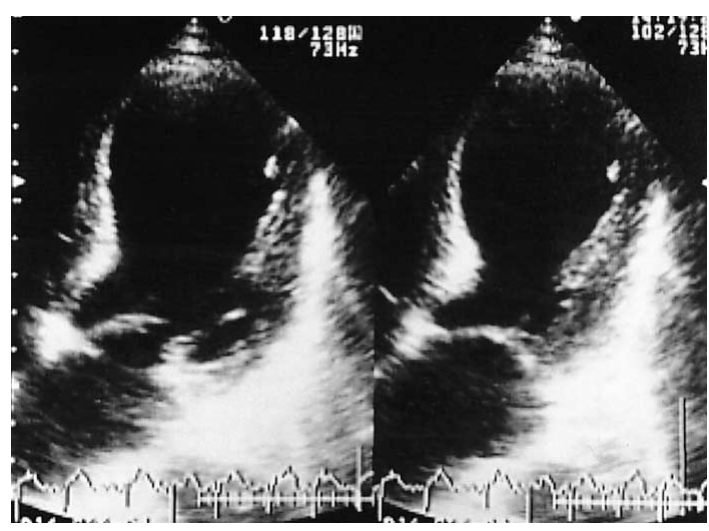

Fig 1. Preoperative echocardiograph showing a left ventricular aneurysm; the anterior to apical wall is thinned and dyskinetic.

Table 1 Changes in the Cardiac Hemodynamics

\begin{tabular}{lccc}
\hline \hline & Preop & After 1 month & After 2 years \\
\hline LVEDVI $\left(\mathrm{ml} / \mathrm{m}^{2}\right)$ & - & 75 & 62 \\
LVEF $(\%)$ & - & 43 & 45 \\
CI $\left(\mathrm{L} / \mathrm{m}^{2}\right)$ & 1.9 & 2.9 & 3.0 \\
PCWP $(\mathrm{mmHg})$ & 27 & 11 & 10 \\
mean PAP $(\mathrm{mmHg})$ & 28 & 19 & 19
\end{tabular}

LVEDVI, left ventricular end-diastolic volume index; LVEF, left ventricular ejection fraction; CI, cardiac index; PCWP, pulmonary capillary wedge pressure; PAP, pulmonary artery pressure. 

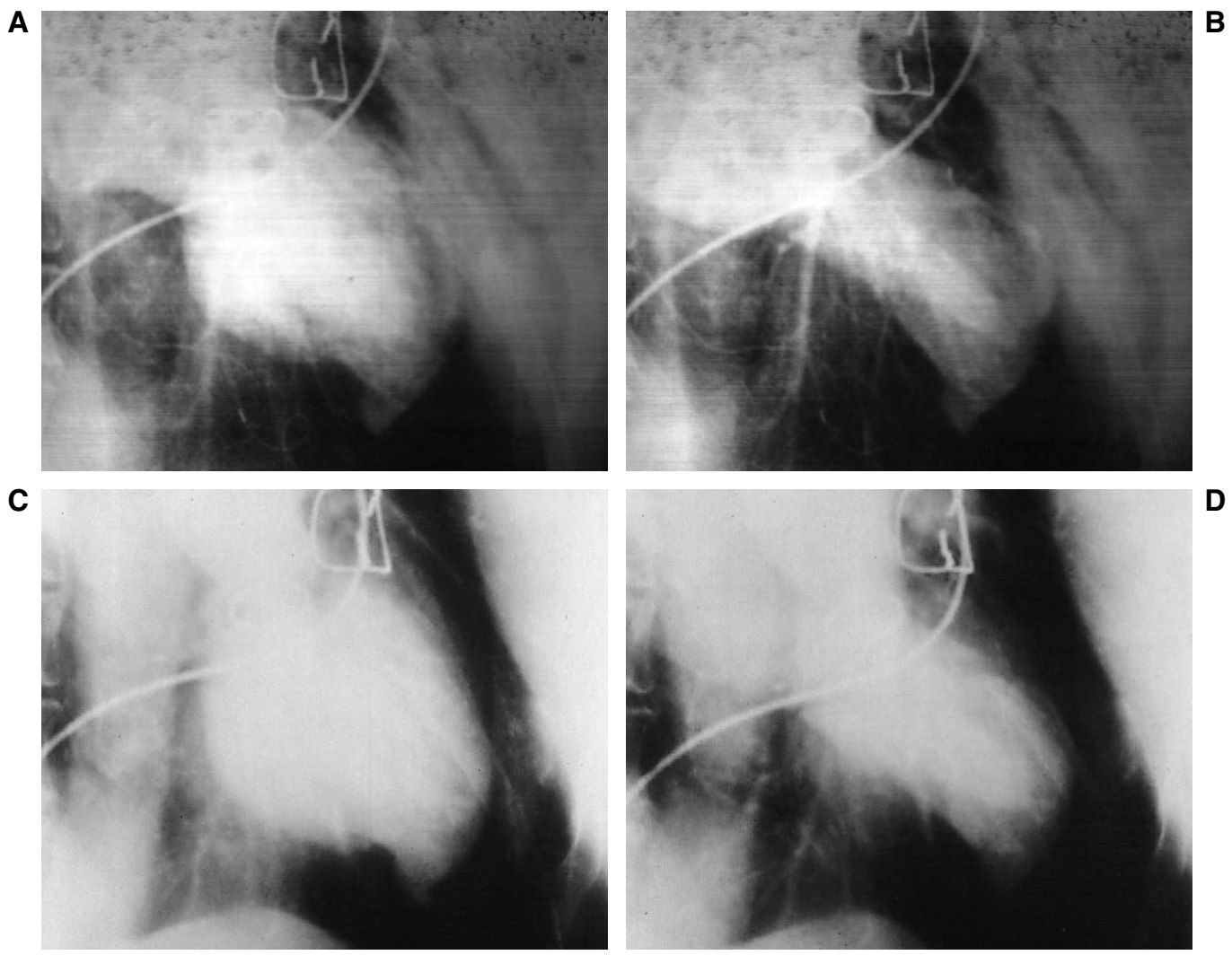

Fig 2. Left ventriculographs showing end-diastolic (A) and end-systolic (B) shape 1 month after the operation, and end-diastolic (C) and end-systolic (D) shape 2 years after the operation.

dial patch was fixed. After closure of the ventriculotomy, tricupsid valve plasty with the DeVega method was done. Weaning from the cardiopulmonary bypass was smooth with the support of moderate doses of catecholamine and intra-aortic balloon pumping. The postoperative clinical course was uneventful. Cardiac catheterization was performed at 1 month (Fig 2A,B) and 2 years after the operation (Fig 2C,D), and both showed satisfactory results (Table 1). At present, the patient is doing well in NYHA functional class I.

\section{Discussion}

Aortic valve stenosis imposes pressure overload on the left ventricular myocardium and leads to myocardial hypertrophy. Therefore, when AMI occurs in patients with AS, the performance of the noninfarcted myocardium continues to impose pressure overload on the infarcted friable tissue, and consequently enhances the occurrence of aneurysm formation or ventricular rupture. Because of these potential mechanisms, AS is an important risk factor for post-infarction cardiac rupture ${ }^{1,2}$ because of the intraventricular hypertension. It is unclear why such catastrophic rupture did not occur in the present case. In a patient with AS and left ventricular aneurysm, the myocardial oxygen efficiency deteriorates during the increase in the left ventricular wall stress and the relative decrease in the coronary blood flow. Because of the cardiac decompensation resulting from this mechanism, the intraventricular pressure probably did not increase extensively, otherwise it would have resulted in catastrophic rupture in the present case. Consequently, the rupture was the oozing type, but there was always the possibility that the oozing rupture could worsen to catastrophic rupture if the operation had been delayed. Therefore, during the preoperative management of AMI with AS, avoid the excessive use of inotropes so as not to increase the intraventricular pressure, thus preserving the peripheral circulation.

In Kadri et al's case, the cardiac rupture was repaired by covering the tear with a pericardial patch, but this procedure can be only hemostatic and exceptional. The standard technique for treatment of the cardiac free wall rupture is linear closure with aneurysmectomy. In Connery's case? the rupture was closed with this technique, but linear closure is still not a physiological repair because it deforms the left ventricular shape postoperatively. Restoration of the left ventricular geometry is important for postoperative left ventricular function,3 so we performed endoventricular circular patch plasty, which reconstructs the physiological shape by creating a neo-apex as well as excluding the friable infarcted area. The patch exclusion of the infarcted area presumably obviates the cardiac remodeling after myocardial infarction, 5 and in fact, there have been no changes in the left ventricular shape or cardiac function throughout the 2-year postoperative follow-up. In the present case, it was unclear whether this operation would reduce the left ventricular volume postoperatively, but our major aim was not volume reduction of the left ventricle, but physiological closure of the cardiac rupture and exclusion of the infarcted area. In addition, we wanted to reduce the intraventricular pressure imposed on the suture line and thereby prevent critical bleeding, as advocated by David and Komeda6

We conclude that not only simple closure of the cardiac 
rupture, but also physiological repair with exclusion of the infarcted area is essential in achieving a good long-term outcome in such a critical case. Special care must be taken with AMI in a patient with AS because of the possible occurrence of aneurysm and rupture of the left ventricle.

\section{References}

1. Kadri MA, Kakadellis J, Campbell CS. Survival after postinfarction cardiac rupture in severe aortic valve stenosis. Eur Heart J 1994; 15: $140-142$.

2. Connery CP, Dumont HJ, Dervan JP, Hartman AR, Anagnostopoulos CE. Transmural myocardial infarction with coexisting critical aortic stenosis as an etiology for early myocardial rupture. $J$ Cardiovasc Surg 1994; 35: 53-56.

3. Jatene AD. Left ventricular aneurysmectomy-resection or reconstruction. J Thorac Cardiovasc Surg 1985; 89: 321-331.

4. Dor V, Saab M, Kornaszewska M, Montiglio F. Left ventricular aneurysm: A new surgical approach. Thorac Cardiovasc Surg 1989; 37: $11-19$.

5. Dor V, Sabatier M, Di Donato M, Montiglio F, Toso A, Maioli M. Efficacy of endoventricular patch plasty in large postinfarction akinetic scar and severe left ventricular dysfunction: Comparison with a series of large dyskinetic scars. J Thorac Cardiovasc Surg 1998; 116: 50-59.

6. Komeda M, David TE, Fremes SE. Surgical repair of postinfarction ventricular septal defect. Circulation; 82(Suppl): IV-243-IV-247. 